\title{
Impact of Spacecraft Shielding on Direct Ionization Soft Error Rates for sub-130 nm Technologies
}

\author{
Jonathan A. Pellish, Member, IEEE, Michael A. Xapsos, Senior Member, IEEE, \\ Craig A. Stauffer, Thomas M. Jordan, Member, IEEE, Anthony B. Sanders, Member, IEEE, \\ Raymond L. Ladbury, Member, IEEE, Timothy R. Oldham, Fellow, IEEE, \\ Paul W. Marshall, Member, IEEE, David F. Heidel, Senior Member, IEEE, \\ and Kenneth P. Rodbell, Senior Member, IEEE
}

\begin{abstract}
We use ray tracing software to model various levels of spacecraft shielding complexity and energy deposition pulse height analysis to study how it affects the direct ionization soft error rate of microelectronic components in space. The analysis incorporates the galactic cosmic ray background, trapped proton, and solar heavy ion environments as well as the October 1989 and July 2000 solar particle events.
\end{abstract}

Index Terms - soft error rate, direct ionization, radiation transport, space environment.

\section{INTRODUCTION}

$\mathrm{S}_{\mathrm{E}}^{\mathrm{o}}$ OLAR activity controls space weather and affects the Earth's atmosphere, including a plasma-physical process called magnetic reconnection, which is the fast release of magnetic energy when oppositely-pointing magnetic field lines are torn apart and reattached to their neighbors. However, we do not understand in depth the physical details of this process. NASA's Magnetospheric MultiScale (MMS) mission [1] is being launched to study magnetic reconnection in Earth's own magnetic field and thereby gain a better understanding of the process in general and specifically how it applies to the production of larger magnetohydrodynamic events like solar flares [2].

To maximize chances of observing reconnection events in Earth's magnetosphere, MMS is being launched during the solar maximum period. Solar maximum, while increasing the probability of gathering good science data, increases the cumulative mission fluence of solar particles incident on the spacecraft, driving up total ionizing dose (TID) as well as rates for soft errors and other single-event effects (SEE) [3-5].

Manuscript received 16 July 2010. This work was supported in part by NASA/GSFC's Internal Research and Development program, the NASA Magnetospheric MultiScale mission, the NASA Electronic Parts and Packaging program, and the Defense Threat Reduction Agency Radiation Hardened Microelectronics program under IACROs \#09-4587I and \#10-4977I to NASA.

J. A. Pellish, M. A. Xapsos, A. B. Sanders, and R. L. Ladbury are with the NASA Goddard Spaceflight Center, 8800 Greenbelt RD, Greenbelt, MD 20771 USA. The corresponding author can be reached at jonathan.a.pellish@nasa.gov.

C. A. Stauffer is with MEI Technologies, 7404 Executive Place STE 500, Seabrook, MD 20706 USA.

T. M. Jordan is with EMPC, PO Box 3191, Gaithersburg, MD 20885.

T. R. Oldham is with Perot Systems Government Services, Inc., c/o NASA/GSFC, Code 561.4, Greenbelt, MD 20771 USA.

P. W. Marshall is a NASA consultant, Brookneal, VA 25428 USA.

D. F. Heidel and K. P. Rodbell are with the IBM T. J. Watson Research Center, Yorktown Heights, NY 10598 USA.

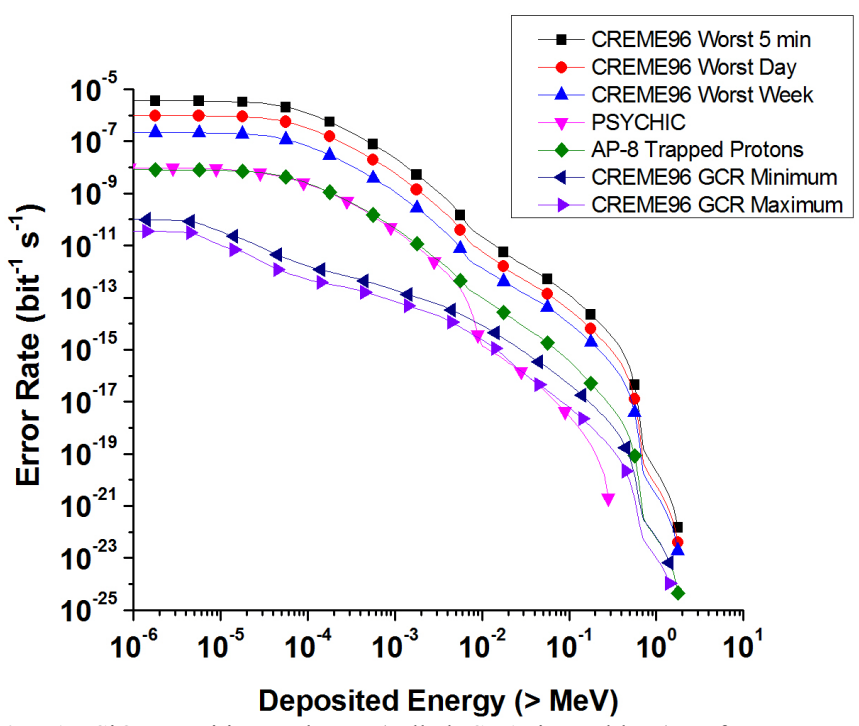

Fig. 1: $\mathrm{SiO}_{2}$ sensitive volume (called $\mathrm{SV} 1$ in Table I) soft error rate relationships for several different space environments behind $2.5 \mathrm{~mm}$ of solid aluminum spherical shielding. All environments are derived from CRÈME96 with the exception of trapped protons and PSYCHIC. The deposited energy becomes the critical energy once a bin is chosen and the rate evaluated. The symbols are sparse to aid viewing - there are 240 bins per trace. The rate curve family for the silicon sensitive volume (called SV2 in Table I) is similar. Note that the PSYCHIC and trapped proton environments are cumulative fluences that were scaled by the inverse of the integration period to convert them to average rates.

Increased TID and SEE rates occur in addition to the everpresent background flux of galactic cosmic rays (GCR) [6], which are also modulated by solar activity.

Although ray trace techniques are commonly used to evaluate complex shielding geometries and obtain TID requirements for space missions, SEE requirements are often determined from simple assumptions about shielding. For example, the Cosmic Ray Effects on Microelectronics code (CRĖME) suite of programs only allows a user the option of doing calculations in solid aluminum sphere geometry with energy deposition based on silicon [7-9]. Due to the increasing sensitivity and complexity - both operational and physical construction - of some devices to SEE, this no longer appears to be adequate. Recent laboratory results show that protons having energies in the vicinity of the Bragg peak can cause soft errors in complementary metal oxide semiconductor (CMOS) technologies at process nodes below $130 \mathrm{~nm}$ [10-15]. This is a serious concern for space missions because protons are the most abundant element in the space environment. A quantitative analysis of the SEE performance of such devices 


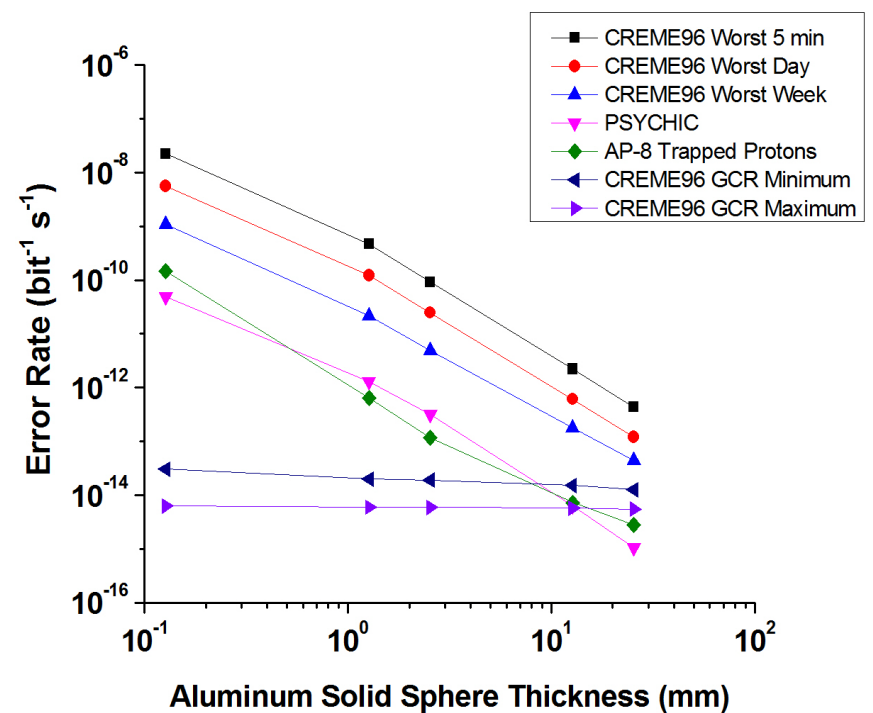

Fig. 2(a): SiO2 SV1 soft error rates for most of the environments in this study with various solid aluminum sphere thicknesses as shielding. The worst $5 \mathrm{~min}$, worst day, worst week, solar minimum, and solar maximum environments are from CREME96. The PSYCHIC environment was calculated at the $95 \%$ confidence level. Note that the PSYCHIC and trapped proton environments are cumulative fluences that were scaled by the inverse of the integration period to convert them to average rates.

in space requires accurate models of the shielding geometry and materials provided by the spacecraft in order to track proton energies down to their end of range and determine if soft errors occur.

While the assumption that SEE rates due to GCR are fairly insensitive to shielding can be justified [7], solar particle events are much more sensitive to shielding. Since the energy spectra of solar event particles is generally softer than those of GCR, use of actual spacecraft shielding in such an analysis can make a significant difference. Recently, a new model of solar particle event energy spectra has been developed [5], which now allows us the opportunity to do this and compare to standard results such as the worst week, worst day, and peak 5 -minute environments in tools like the CRÈME code [7, 16] and the Space Environment Information System (SPENVIS) [17]. Furthermore, we use these same tool sets to develop an environmental model of the July 2000 "Bastille Day" solar event so that a comparison can be made to the October 1989 event. The October 1989 storm has been the de facto worstcase environment since the release of CRÈME96.

We focused this work on evaluating environment-specific soft error rates in a volatile and non-volatile memory technology arising from various shielding distributions: solid aluminum spheres of different thicknesses, an aluminum cube with $2.5 \mathrm{~mm}$ walls, an isolated spacecraft electronics box, and that electronics box embedded in an actual spacecraft. The soft error rates in these memories are dominated by direct ionization effects $[12,18,19]$. We chose environments consistent with an Earth-based satellite with an highlyelliptical orbit, including trapped protons, Prediction of Solar particle Yields for CHaracterizing Integrated Circuits (PSYCHIC)-based solar heavy ions [4, 5], as well as GCR and two worst-case solar particle events based on the October 1989 and July 2000 storms [7]. The soft error rates are calculated with NOVICE [20, 21], using strict adjoint numerical integration techniques. These analyses will enable

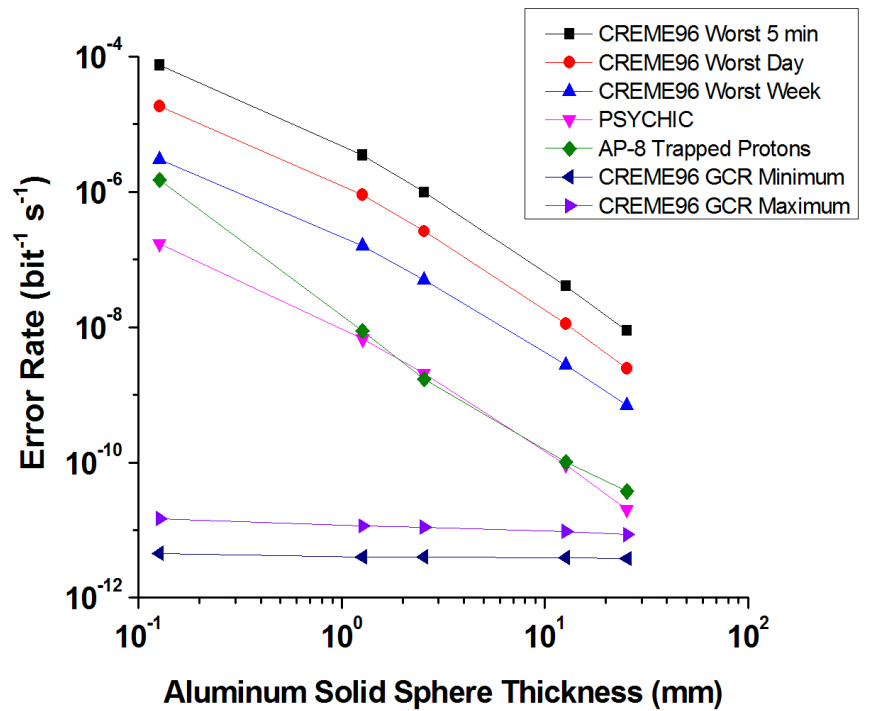

Fig. 2(b): Silicon SV2 soft error rates for most of the environments in this study with various solid aluminum sphere thicknesses as shielding. The worst 5 min, worst day, worst week, solar minimum, and solar maximum environments are from CRÈME96. The PSYCHIC environment was calculated at the $95 \%$ confidence level. Note that the PSYCHIC and trapped proton environments are cumulative fluences that were scaled by the inverse of the integration period to convert them to average rates.

insightful comparisons and improve risk mitigation for NASA-relevant commercial technologies destined for flight project insertion.

Table I: Sensitive Volume Parameters

\begin{tabular}{cccccc}
\hline $\begin{array}{c}\text { Sensitive } \\
\text { Volume }\end{array}$ & $\begin{array}{c}\mathrm{X} \\
(\mathbf{n m})\end{array}$ & $\begin{array}{c}\mathrm{Y} \\
(\mathbf{n m})\end{array}$ & $\begin{array}{c}\mathrm{Z} \\
(\mathbf{n m})\end{array}$ & $\begin{array}{c}\mathrm{Q}_{\text {crit }} \\
\mathbf{( f C})\end{array}$ & $\begin{array}{c}\mathrm{E}_{\text {crit }} \\
(\mathbf{k e V})\end{array}$ \\
\hline $\begin{array}{c}\text { SV1 } \\
\left(\mathrm{SiO}_{2}\right)\end{array}$ & & & & & \\
$\mathbf{4}$ Gbit SLC & 63 & 50 & 10 & 0.06 & 6.6 \\
$\begin{array}{c}\text { NAND Flash } \\
\quad \text { SV2 } \\
\text { (Silicon) } \\
\text { 45 nm SOI } \\
\text { SRAM }\end{array}$ & 450 & 450 & 100 & 0.5 & 11 \\
\hline
\end{tabular}

\section{SENSITIVE Volume DesCRIPTIONS}

We chose two sensitive volumes for soft error rate calculation comparison across the different environments and shielding distributions examined in this work. Their parameters are shown in Table I, where $Q_{\text {crit }}$ is the critical charge and $E_{\text {crit }}$ is the critical energy, implying that meeting or exceeding either one within the sensitive volume will result in a soft error. These volumes, while based on very real technologies, were chosen as contrasting examples. While their general behavior can be related back to the technology in question, this work is not meant to compute applicationspecific soft error rates for the devices under consideration.

The first volume $\left(\mathrm{SV} 1, \mathrm{SiO}_{2}\right)$ is based on the oxide charge storage stack in the Samsung K9F4G08U0A 4 Gbit singlelevel cell NAND flash memory, which is defined based on process reverse engineering [22]. The mechanisms proposed to explain soft errors in the floating gate are connected with the phenomena of charge loss from the floating gate due to a transient conductive path across the tunnel oxide as well as charge trapping in the tunnel oxide [18, 23-25]. The heavy ion 


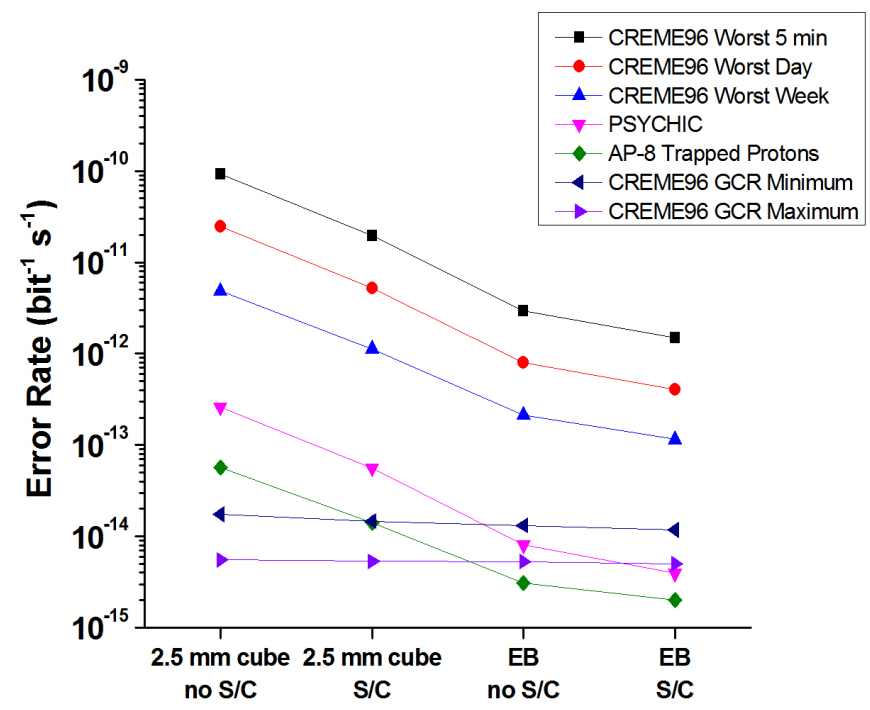

Fig. 3(a): $\mathrm{SiO}_{2} \mathrm{SV} 1$ soft error rates for most of the environments in this study. However, these rates consider several more realistic shielding configurations. A cube with $2.5 \mathrm{~mm}$ aluminum walls and an actual electronics box (EB) are simulated with and without a complete spacecraft $(\mathrm{S} / \mathrm{C})$. The cube is placed within the spacecraft such that its shielding environment is similar to the electronics box. The PSYCHIC environment was calculated at the $95 \%$ confidence level. Note that the PSYCHIC and trapped proton environments are cumulative fluences that were scaled by the inverse of the integration period to convert them to average rates.

sensitivity of this structure was determined by accelerated ground testing, c.f. Fig. 3b in [19]. We calculated the critical charge based on a $\mathrm{SiO}_{2}$ electron-hole pair creation energy of $17 \mathrm{eV}$, which translates to $106 \mathrm{keV} / \mathrm{fC}[26,27]$. The second volume (SV2, silicon) is derived from the $45 \mathrm{~nm}$ silicon on insulator (SOI) static random access memory (SRAM) data presented by D. F. Heidel, et al. [12]. Its dimensions were derived by taking the square root of the saturated heavy ion cross section in Fig. 2 of [12] and assuming a $100 \mathrm{~nm}$ silicon body thickness. The body thickness is a conservative engineering estimate; the reader should not assume it is specific to the example technology used here. The critical charge is based on the electron-hole pair creation energy in silicon of $3.6 \mathrm{eV}[28,29]$. No further technology information was used.

We reached these conclusions regarding the sensitive volume dimensions and critical upset parameters based on publicly available information and several conservative assumptions. These parameter estimates are not meant to be definitive, but as a means of comparing the soft error rates of different environments and shielding configurations given a well-defined definition of upset in two distinct technologies.

\section{Soft ERRor RATE CALCULATIONS}

\section{A. NOVICE Methodology}

The NOVICE soft error rate calculations produce a pulse height spectrum that is reverse-integrated to produce a curve of event counts in a sensitive volume over a given time interval versus energy deposited. The soft error rate can then be determined once the critical energy is known. An example of these soft error rate curves for SV1 is shown in Fig. 1. Based on the information in Table I, for the CRÈME96 GCR solar maximum, the 4 Gbit NAND flash would have rate of

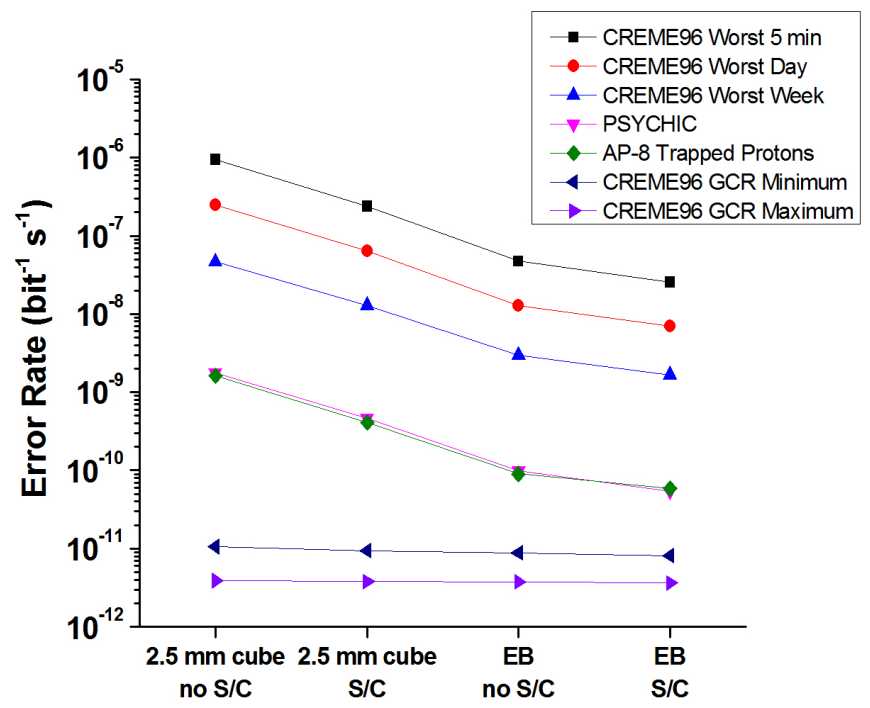

Fig. 3(b): Silicon SV2 soft error rates for most of the environments in this study. However, these rates consider several more realistic shielding configurations. A cube with $2.5 \mathrm{~mm}$ aluminum walls and an actual electronics box (EB) are simulated with and without a complete spacecraft $(\mathrm{S} / \mathrm{C})$. The cube is placed within the spacecraft such that its shielding environment is similar to the electronics box. The PSYCHIC environment was calculated at the $95 \%$ confidence level. Note that the PSYCHIC and trapped proton environments are cumulative fluences that were scaled by the inverse of the integration period to convert them to average rates.

$2.4 \times 10^{-5}$ errors $/ \mathrm{s}$ behind $2.5 \mathrm{~mm}$ of solid spherical aluminum shielding. For GCR environments based on CRÈME96, the flux versus energy spectra for solar maximum and solar minimum represent the extreme points of the solar cycle and vary slowly.

Aside from GCR environments, we also performed rate calculations for cumulative solar maximum solar heavy ions and trapped protons. The trapped proton spectra were compiled as total fluences over 2.5 years based on the AP-8 model. The proton transport and rate calculations are unique from the other calculations in that they include elastic recoils from the surrounding material as well as the sensitive volume. The elastic recoils roughly double the pulse height counts in the larger energy bins and have the ability to extend the energy deposition distribution. We also computed the PSYCHIC solar heavy ion environment fluences at the $95 \%$ confidence level for one solar active year, which includes all naturallyoccurring elements in the periodic table. The major components are $\mathrm{H}, \mathrm{He}, \mathrm{C}, \mathrm{N}, \mathrm{O}, \mathrm{Ne}, \mathrm{Mg}, \mathrm{Si}, \mathrm{S}$, and $\mathrm{Fe}$ ions. The fluences for these solar heavy ions are based on data from the Interplanetary Monitoring Platform (IMP-8) Goddard Medium Energy (GME) experiment [30] and the Advanced Composition Explorer (ACE) Solar Isotope Spectrometer (SIS) [31] instruments. The trapped proton and PSYCHIC solar heavy ion environment models produce long-term averages that have been scaled by the inverse of the integration period in order to calculate an average rate per second.

We have made some fundamental assumptions about the soft error rates presented here. In the case of both sensitive volumes, we assumed a monolithic cross section, which is physically impossible. This is necessary in the absence of fundamental knowledge about the process, physical layout, and soft error mechanisms. An experiment with CRÈME96 
shows that increasing the number of bits per device while conserving the total area of the original monolithic volume decreases the soft error rate. Using the parameters in Table I and splitting the volume into eight "bits" with $x y$-dimensions of $0.159 \mu \mathrm{m}$ per side with the same thickness showed a decrease of approximately $7 \%$ in the geostationary GCR error rate behind $2.54 \mathrm{~mm}$ of aluminum shielding. This is a simple quantitative example and cannot necessarily be extrapolated to other scenarios, but it does highlight a relevant trend as well as illuminate one of the caveats in CRÈME96. Regardless, the most significant issue with using a monolithic sensitive volume is that it does not permit evaluation of multi-cell effects, which are an obvious concern for sub-130 nm technologies. While Heidel published general information about the SRAM cell structure for different bit patterns [12], the description is insufficient for making drastic modifications to the current setup. However, these topics are outside the scope of this paper. Actually, NOVICE can do calculations with multiple sensitive volumes, but it has not been rigorously verified.

Secondly, we neglected nuclear inelastic reactions, assuming that the observable soft error rate is driven by direct ionization of the primary particle, which could be a proton or heavy ion, and ionization caused by proton-based elastic reactions $[10-15,32]$. Proton direct ionization was initially observed in a $65 \mathrm{~nm}$ SOI technology, reported by Rodbell et $a l$. in 2007. The conclusion was based on experimental data from a 3 MV Tandem Van de Graaff accelerator with excellent energy resolution in addition to intimate process knowledge and circuit simulations. Subsequent investigations recorded similar results in $90 \mathrm{~nm}$ bulk, $65 \mathrm{~nm}$ bulk, and $45 \mathrm{~nm}$ SOI processes.

Furthermore, in the critical energy regime of these devices, the mechanism assumption above is likely valid for single-bit soft errors. Heidel showed that multiple-cell cross sections, specifically double-bit cross sections, increased at low proton energies indicating a direct linkage to proton direct ionization [12]. The mechanism specified in the paper was elastic scattering since no other conclusion seemed likely for normal incidence, low-energy irradiations. This effect was tightly coupled to the layout of the SRAM cell and may not necessarily be observed in other technologies. The NOVICE calculations completed for this paper do include elastic scattering effects for trapped protons; however, no multi-cell effects were modeled.

Finally, the calculations presented here do not have their uncertainties evaluated, which would be a challenging undertaking. The errors inherent in these calculations are systematic and based on assumptions related to the sensitive volumes and the space environment. An upper bound for the PSYCHIC solar heavy ion rate is possible since it is based on an underlying technique that provides confidence levels. For instance, the long term solar heavy ion fluences for solar active years used in this paper were calculated at the $95 \%$ confidence level. The AP-8 and CRÈME96 models do not have this feature. It is important to note that the space environment is dynamic and long term particle flux/fluence may not accurately describe short term or instantaneous conditions at a given point in space.

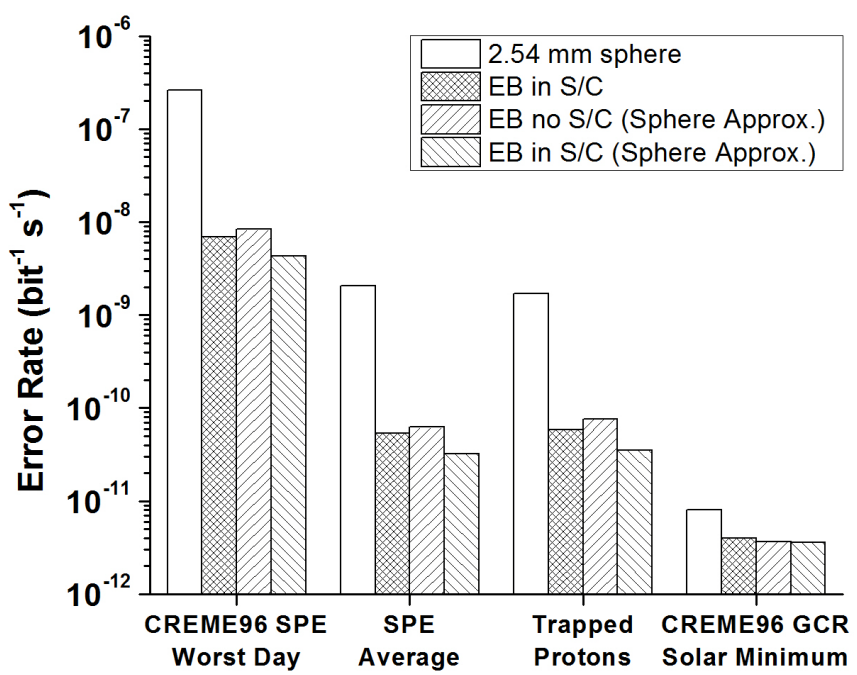

Fig. 4: Silicon SV2 comparison of environmental soft error rates for the standard $2.5 \mathrm{~mm}$ solid spherical aluminum shield and an electronics box (EB) within an actual spacecraft. Also shown are the rates for solid spherical approximations of the electronics box both with and without the spacecraft. These sphere thicknesses are based on the average shield thicknesses shown in Table II $-15.9 \mathrm{~mm}$ and $23.4 \mathrm{~mm}$. Between the $2.5 \mathrm{~mm}$ solid sphere shield and the electronics box within the spacecraft, both the worst day and the solar particle event average differ by a factor of 38 , trapped protons by a factor of 28 , and GCR solar minimum by a factor of 2 . These differences highlight the conservative nature of small-thickness spherical shielding. Similar results are observed for the $\mathrm{SiO}_{2} \mathrm{SV} 1$.

\section{B. Solid Aluminum Spherical Shielding}

The combined plots showing the error rates for all seven environments for both sensitive volumes are shown in Figs. 2(a) and 2(b). The worst case solar particle event (SPE) environments from CRÈME96 - worst five minutes, worst day, and worst week - produce the highest rates for both SV1 and SV2. The CRÈME96 solar minimum and maximum GCR environments are not appreciably affected by increased shielding. However, shielding impacts the SPE environments, trapped protons, and PSYCHIC solar heavy ions producing large variations in soft error rates for a given shielding thickness. The soft error rate from solar heavy ion and trapped proton spectra direct ionization dominates the ambient space environment for both SV1 and SV2, though shielding can mitigate the proton rate past $10 \mathrm{~mm}$ of aluminum in the case of SV1. The trapped protons and solar heavy ions dominate the error rate of SV2 outside of SPE conditions up to and past $25.4 \mathrm{~mm}$ (1 in) of solid spherical aluminum shielding.

As a comparison to CRÈME96, several calculation points have been made for SV2. We cannot compare CRÈME96 with the NAND flash (SV1) rate calculations because the sensitive volume is $\mathrm{SiO}_{2}$, which is not possible in the CRÈME96 framework. This is possible using the expanded features in CRÈME-MC. Using the $Q_{\text {crit }}$ heavy ion upset prediction tool, we calculated geosynchronous solar maximum and minimum rates. The CRĖME96 rates for SV2 in solar maximum and minimum are $2.3 \times 10^{-12}$ errors/(bit $\left.\cdot \mathrm{s}\right)$ and $6.3 \times 10^{-12}$ errors/(bit $\cdot \mathrm{s}$ ) assuming $2.5 \mathrm{~mm}$ of aluminum shielding. These rates are within a factor of two of the corresponding NOVICE rates of $3.5 \times 10^{-12}$ errors/(bit.s) and $9.8 \times 10^{-12}$ errors/(bit $\left.\cdot \mathrm{s}\right)$. This level of agreement is encouraging, bearing in mind that NOVICE is actually transporting the particle flux-energy spectra through materials, including the sensitive volume, 
whereas CRÈME96 is combining path lengths with a linear energy transfer spectrum in the sensitive volume.

\section{Spacecraft Shielding}

Radiation engineers and scientists use solid spherical shielding in the absence of more detailed mechanical models or because use of such models is outside the scope of work. Standard practice assumes $2.5 \mathrm{~mm}$ - roughly $100 \mathrm{mil}$ - of solid spherical aluminum to provide uniform $4 \pi$ sr coverage. However, detailed analysis of actual spacecraft electronics boxes and superstructure generally shows that $2.5 \mathrm{~mm}$ of shielding is an underestimation. In truth, many electronics boxes have walls that are thicker than $2.5 \mathrm{~mm}$, and that does not consider the additional mass of the surrounding spacecraft. Furthermore, rectangular parallelepipeds do not provide uniform shielding over $4 \pi$ sr like spherical shielding. Particles incident on a sensitive volume just inside a rectangular box surface must travel through significantly increasing shielding as the angle of incidence measured normally from the surface increases.

Table II: Electronics Box NOVICE Statistics for 16500 Rays (762 $\mu \mathrm{sr} /$ ray)

\begin{tabular}{lccc}
\hline \multicolumn{1}{c}{ Box Type } & $\begin{array}{c}\text { Minimum } \\
\text { (mm) }\end{array}$ & $\begin{array}{c}\text { Maximum } \\
\text { (mm) }\end{array}$ & $\begin{array}{c}\text { Average } \\
\text { (mm) }\end{array}$ \\
\hline Cube, no S/C & 2.54 & 4.34 & 2.97 \\
Cube, in S/C & 2.62 & 1810 & 4.75 \\
$\begin{array}{l}\text { Electronics box, } \\
\text { no S/C }\end{array}$ & 4.45 & 108 & 15.9 \\
$\begin{array}{l}\text { Electronics box, } \\
\text { in S/C }\end{array}$ & 4.45 & 1620 & 23.4 \\
\hline
\end{tabular}

The spacecraft used in this study has a dry mass of approximately $800 \mathrm{~kg}$, is octagonal in shape, has decks enclosing the top and bottom faces, and is wrapped in its solar panels. The electronics box used is the central instrument data processor box, which is part of the command and data handling infrastructure. This box is made of aluminum and is located inside the spacecraft, approximately half way between the top and bottom decks. Table II shows the ray path statistics for a cube with $2.5 \mathrm{~mm}$ walls and the central instrument data processor electronics box.

Figs. 3(a) and 3(b) show soft error rates for SV1 and SV2 in various realistic configurations that do not rely on spherical shielding. As expected, the solar particle event and trapped proton environments are more sensitive to the amount of shielding than GCR. These shielding configurations also demonstrate the same behavior as the solid spherical shielding in Figs. 2(a) and 2(b) in that the large amount of shielding around SV1 eventually blocks out the contributions from trapped protons and solar particles, leaving GCR to dominate the soft error rate. SV2 is always dominated by trapped protons and solar particles. It is important to note that the complete electronics box provides the largest amount of independent shielding - more than the generic cube within the spacecraft and more than the spacecraft itself. The critical point is that the average ray path thickness of the electronics box inside the spacecraft is larger by a factor of five relative to the average ray path thickness of the $2.5 \mathrm{~mm}$-walled cube inside the spacecraft.
While isolated, perhaps these comparisons between different shielding environments do not seem significant. However, as shown in Fig. 4, the difference is more than a factor of 25 between the complete electronics box inside the spacecraft and $2.5 \mathrm{~mm}$ of solid spherical shielding for all but the GCR solar minimum environment. For further comparison, rates for solid spherical shielding based on the average ray trace shielding depths in Table II are shown in Fig. 4. The rates were determined by fitting a power law curve to the points in Fig. 2(b) and then evaluating the equations at $15.9 \mathrm{~mm}$ and $23.4 \mathrm{~mm}$. The average shielding depth, when converted to a solid sphere, appears to underestimate the rate for the electronics box within the spacecraft and overestimate the rate for the bare box without the spacecraft. However, the rates using solid spherical shielding approximating just the box bound the more complex problem of computing the soft error rate for the box and the spacecraft.

\section{Examination of the July 2000 Event}

Throughout this investigation, we have only considered the October 1989 solar particle event in terms of worst case environments. It is known to radiation effects engineers as the worst week, worst day, and worst five minutes selections in CRÈME96 and CRÈME-MC tool suites. The solar maximum period following the one that produced the October 1989 storm produced another very large solar particle event in July 2000. The particle fluxes for the July 2000 event remained above background for more than one month. However, the highest fluxes by far, particularly for heavy ions, occurred over a period of a few days shortly after the event onset. Previous measurements of LET spectra by C.S. Dyer et al. have shown that this event and two others during solar cycle 23 approximately equaled the "worst day" model in CRÈME96 [33]. Thus, further investigation is warranted. We have therefore analyzed the IMP-8 GME and ACE SIS data for the worst two day period of the July 2000 event to obtain energy spectra of the 10 most abundant elements as outlined in [5]. Other satellite data and an abundance model were used to determine the relatively small contributions of the remaining naturally occurring elements in the periodic table, again as in [5]. Since the worst two-day period was used, the number of calculated upsets were divided by 2 before comparison to the upsets produced by the worst day portion of the October 1989 event in the CRĖME models. A period of 2 days was chosen because the fluxes of different ions peak at different times during this period and it was not clear that initially limiting our analysis to 24 hours would be "worst case" for both memories that we consider.

Figs. 5(a) and 5(b) show SV1 and SV2 soft error rates for the October 1989 and July 2000 worst days for the cube with $2.5 \mathrm{~mm}$ walls and the electronics box, both with and without the spacecraft. SV1 produces similar results for both events and is insensitive to any differences in event composition. SV2 yields higher soft error rates for the October 1989 worst day environment, though the increase over July 2000 is only a factor of two. We conclude that the two events are similar given our stated geometries and upset sensitivities. 


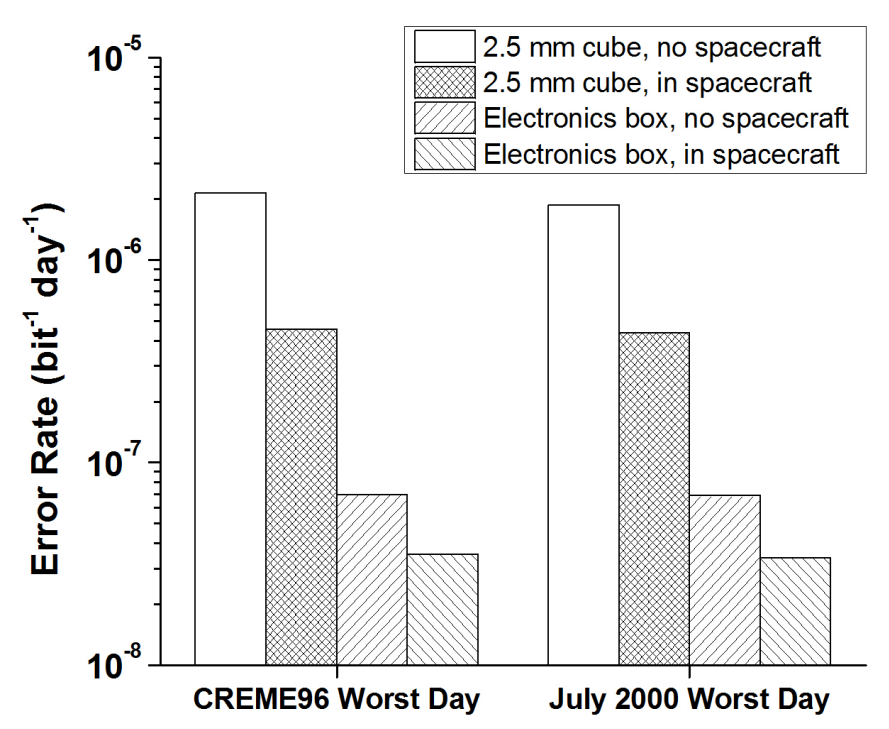

Fig. 5(a): $\mathrm{SiO}_{2} \mathrm{SV} 1$ soft error rate comparison for the worst day during the past two solar maximum periods - the October 1989 event and the July 2000 event. The cube, electronics box, and spacecraft are the same as those discussed earlier.

\section{DISCUSSION}

The soft error rate calculations presented in Section III, while for simple shielding geometries, reveal interesting trends and highlight issues necessary for spacecraft mission assurance. The two most critical aspects of the simulation results presented in Figs. 2 and 3 are the contributions from solar heavy ions and trapped protons, recalling that NOVICE only computed direct ionization of the primary particle and any elastic recoils produced during the trapped proton computations. We showed that the October 1989 SPE is still sufficient for worst case analysis, but the margin available for a given application could result in either over- or underestimation of the soft error risk, yielding higher costs for unintentional over-engineering, redesign, or anomaly resolution. These conclusions apply to both SV1 and SV2.

In the past, with larger, less sensitive technologies, the effects of protons were limited to TID, displacement damage effects, and single-event effects related to indirect ionization. However, as commercial vendors scale bulk CMOS and SOI technologies, direct ionization proton events have become reality [11-13]. This conclusion is presently only applicable to SV2, the silicon volume based on an SOI SRAM. While we demonstrated that a $\mathrm{SiO}_{2}$ volume with the characteristics of SV1 would be susceptible to the described proton and heavy ion direct ionization effects, the proton effects have not been experimentally observed for NAND flash technology to date. A future flash or charge trap technology may show different behavior. A large portion of the weight in the solar particle and trapped proton rates considered here is due to direct ionization, which, given their abundance, drives up the soft error rate. We did not intentionally focus this work on lowenergy proton rate calculations, but the effect is substantial for the geometries and sensitivities chosen here. Note that for $\mathrm{SV} 1$, the error rates for trapped protons and the long-term solar particle environment are brought down to a comparable level with GCR solar maximum through the use of the heavily shielded electronics box. On the other hand, for SV2 they

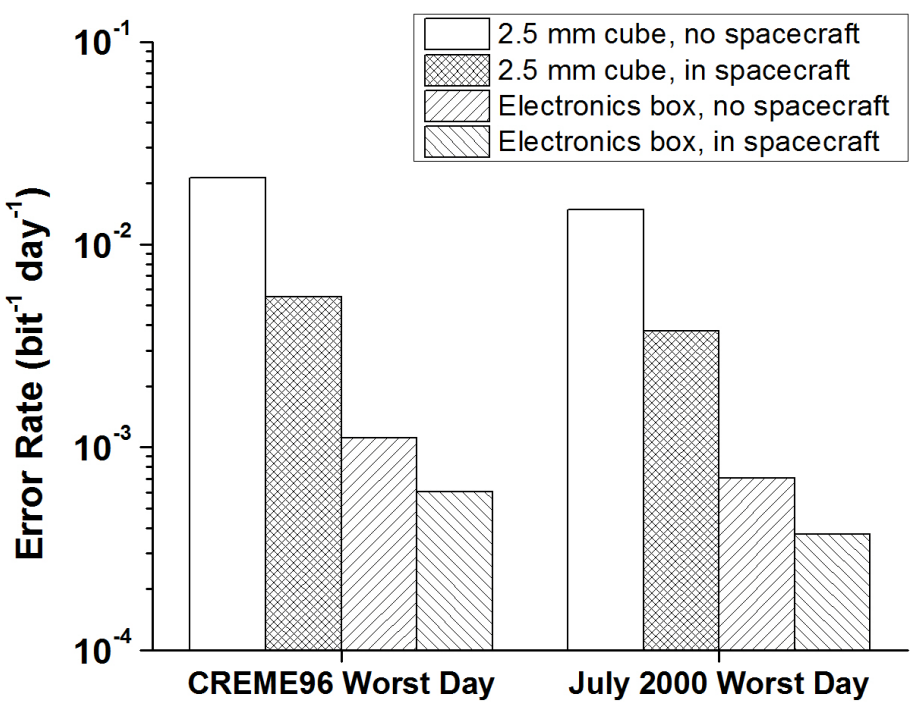

Fig. 5(b): Silicon SV2 soft error rate comparison for the worst day during the past two solar maximum periods - the October 1989 event and the July 2000 event. The cube, electronics box, and spacecraft are the same as those discussed earlier.

remain well above the GCR rates. While the critical energies for SV1 and SV2 are within a factor of two, the electron-hole pair creation energy is different and the size of SV1 is much smaller. In fact, the volume of SV2 is over six hundred times larger than the volume of SV1, making it easier to deposit the critical energy.

We note the substantial contribution from solar heavy ions, which equals or exceeds the GCR contribution during the solar maximum period. This applies to both SV1 and SV2 sensitive volumes. Traditionally, average solar heavy ion fluences are not modeled when doing basic rate calculations, but this work suggests that they are important with sensitive technologies. The PSYCHIC solar heavy ion model also includes proton fluences as calculated in the Emission of Solar Protons (ESP) model [3-5], and is therefore a confidence level based approach. In the future, when evaluating soft error rates for sensitive technologies, it may be necessary to incorporate solar heavy ions on a regular basis when mission durations fall within solar active years.

\section{CONCLUSIONS}

We have demonstrated that the use of simplified assumptions of solid sphere shielding generally overestimate soft error rates due to direct ionization. This can be especially important in modern commercial devices with low upset thresholds and scaled geometries. Using solid sphere shielding assumptions may lead to overdesign and increased mission cost.

For the sensitive volume examples considered here, the soft error rates were overestimated by about $2 \mathrm{x}$ for GCR during solar minimum, $28 \mathrm{x}$ for the long-term solar particle and trapped proton environments, and $38 \mathrm{x}$ for the CRÈME96 worst day. This is analogous to TID and displacement damage dose requirement trends with shielding analysis. For the two memories considered, the trapped proton environment, not the widely quoted GCR environment, always contributed 
substantially and often dominated the soft error rates under ambient conditions. To better understand this effect, more shielding configurations and orbits should be analyzed. In addition to trapped protons, the solar particle environment can also cause more soft errors than GCR over the long-term during solar maximum periods.

Due to the increasing importance of shielding analysis for SEE, tools like NOVICE and Geant4-based applications (CRÈME-MC and SPENVIS/MULASSIS) are becoming necessities. These tools need to be validated with space data.

\section{ACKNOWLEDGEMENT}

The authors would like to thank the MMS project staff and engineers, Ken LaBel, Lew Cohn, Bruce Wilson, Maj. Eric Heigel (USAF), and Lt. Col. Warren Nuibe (USAF) for their continued support of this work.

\section{REFERENCES}

[1] STP Magnetospheric MultiScale Mission. Available: http://stp.gsfc.nasa.gov/missions/mms/mms.htm; http://mms.space.swri.edu/

[2] J. L. Burch and J. F. Drake, "Reconnecting magnetic fields," American Scientist, vol. 97, no. 5, pp. 392399, Sep-Oct 2009.

[3] M. A. Xapsos, G. P. Summers, J. L. Barth, E. G. Stassinopoulos, and E. A. Burke, "Probability model for cumulative solar proton event fluences," IEEE Trans. Nucl. Sci., vol. 47, no. 3, pp. 486-490, Jun. 2000.

[4] M. A. Xapsos, C. Stauffer, G. B. Gee, J. L. Barth, E. G. Stassinopoulos, and R. E. McGuire, "Model for solar proton risk assessment," IEEE Trans. Nucl. Sci., vol. 51, no. 6, pp. 3394-3398, Dec. 2004.

[5] M. A. Xapsos, C. Stauffer, T. Jordan, J. L. Barth, and R. A. Mewaldt, "Model for cumulative solar heavy ion energy and linear energy transfer spectra," IEEE Trans. Nucl. Sci., vol. 54, no. 6, pp. 1985-1989, Dec. 2007.

[6] J. Feynman and S. B. Gabriel, "High-energy charged particles in space at one astronomical unit," IEEE Trans. Nucl. Sci., vol. 43, no. 2, pp. 344-352, Apr. 1996.

[7] A. J. Tylka, J. H. Adams Jr, P. R. Boberg, B. Brownstein, W. F. Dietrich, E. O. Flueckiger, E. L. Petersen, M. A. Shea, D. F. Smart, and E. C. Smith, "CREME96: a revision of the Cosmic Ray Effects on Micro-Electronics Code," IEEE Trans. Nucl. Sci., vol. 44, no. 6, pp. 2150-2160, Dec. 1997.

[8] R. A. Weller, R. A. Reed, K. M. Warren, M. H. Mendenhall, B. D. Sierawski, R. D. Schrimpf, and L. W. Massengill, "General framework for single event effects rate prediction in microelectronics," IEEE Trans. Nucl. Sci., vol. 56, no. 6, pp. 3098-3108, Dec. 2009.

[9] CREME96: Cosmic Ray Effects on Microelectronics. Available: https://creme96.nrl.navy.mil/

[10] K. P. Rodbell, D. F. Heidel, H. H. K. Tang, M. S. Gordon, P. Oldiges, and C. E. Murray, "Low-Energy Proton-Induced Single-Event-Upsets in $65 \mathrm{~nm}$ node,
Silicon-on-Insulator, Latches and Memory Cells," vol. 54, no. 6, pp. 2474-2479, Dec. 2007.

[11] D. F. Heidel, P. W. Marshall, K. A. LaBel, J. R. Schwank, K. P. Rodbell, M. C. Hakey, M. D. Berg, P. E. Dodd, M. R. Friendlich, A. D. Phan, C. M. Seidleck, M. R. Shaneyfelt, and M. A. Xapsos, "Low energy proton single-event upset test results on 65 nm SOI SRAM," IEEE Trans. Nucl. Sci., vol. 55, no. 6, pp. 3394-3400, Dec. 2008.

[12] D. F. Heidel, P. W. Marshall, J. A. Pellish, K. P. Rodbell, K. A. LaBel, J. R. Schwank, S. E. Rauch, M. C. Hakey, M. D. Berg, C. M. Castaneda, P. E. Dodd, M. R. Friendlich, A. D. Phan, C. M. Seidleck, M. R. Shaneyfelt, and M. A. Xapsos, "Single-event upsets and multiple-bit upsets on a $45 \mathrm{~nm}$ SOI SRAM," IEEE Trans. Nucl. Sci., vol. 56, no. 6, pp. 3499-3504, Dec. 2009.

[13] B. D. Sierawski, J. A. Pellish, R. A. Reed, R. D. Schrimpf, K. M. Warren, R. A. Weller, M. H. Mendenhall, J. D. Black, A. D. Tipton, M. A. Xapsos, R. C. Baumann, D. Xiaowei, M. J. Campola, M. R. Friendlich, H. S. Kim, A. M. Phan, and C. M. Seidleck, "Impact of low-energy proton induced upsets on test methods and rate predictions," IEEE Trans. Nucl. Sci., vol. 56, no. 6, pp. 3085-3092, Dec. 2009.

[14] E. H. Cannon, M. Cabanas-Holmen, J. Wert, T. Amort, R. Brees, J. Koehn, B. Meaker, and E. Normand, "Heavy ion, high energy and low energy proton SEE sensitivity of 90-nm RHBD SRAMs," presented at the IEEE Nuclear and Space Radiation Effects Conf., Denver, CO, 2010.

[15] N. Haddad, A. Kelly, R. Lawrence, B. Li, J. Rodgers, and J. Ross, "Heavy ion, high energy and low energy proton SEE sensitivity of 90-nm RHBD SRAMs," presented at the 11th European Conf. on Radiation and Its Effects on Components and Systems, Langenfeld, Austria, 2010.

[16] CREME-MC: CREME Monte Carlo. Available: https://creme-mc.isde.vanderbilt.edu/

[17] SPENVIS: The Space Environment Information System. Available: http://www.spenvis.oma.be/

[18] T. R. Oldham, R. L. Ladbury, M. Friendlich, H. S. Kim, M. D. Berg, T. L. Irwin, C. Seidleck, and K. A. LaBel, "SEE and TID characterization of an advanced commercial 2 Gbit NAND flash nonvolatile memory," IEEE Trans. Nucl. Sci., vol. 53, no. 6, pp. 3217-3222, Dec. 2006.

[19] T. R. Oldham, M. R. Friendlich, A. B. Sanders, C. M. Seidleck, H. S. Kim, M. D. Berg, and K. A. LaBel, "TID and SEE response of advanced Samsung and Micron 4G NAND flash memories for the NASA MMS mission," in IEEE Radiation Effects Data Workshop, Quebec City, Quebec Canada, 2009, pp. 114-122.

[20] T. M. Jordan, "An adjoint charged particle transport method," IEEE Trans. Nucl. Sci., vol. 23, no. 6, pp. 1857-1861, Dec. 1976.

[21] T. M. Jordan, "The accuracy of NOVICE electron shielding calculations," in 1st European Conf. on 
Radiation and Its Effects on Components and Systems, La Grande-Motte, France, 1991, pp. 320324.

[22] D. James, "Nano-scale flash in the mid-decade," in Advanced Semiconductor Manufacturing Conf., Stresa, Italy, 2007, pp. 371-376.

[23] G. Cellere, P. Pellati, A. Chimenton, J. Wyss, A. Modelli, L. Larcher, and A. Paccagnella, "Radiation effects on floating-gate memory cells," IEEE Trans. Nucl. Sci., vol. 48, no. 6, pp. 2222-2228, Dec. 2001.

[24] S. M. Guertin, D. M. Nguyen, and J. D. Patterson, "Microdose induced data loss on floating gate memories," IEEE Trans. Nucl. Sci., vol. 53, no. 6, pp. 3518-3524, Dec. 2006.

[25] M. Bagatin, S. Gerardin, G. Cellere, A. Paccagnella, A. Visconti, S. Beltrami, M. Bonanomi, and R. Harboe-Sorensen, "Annealing of heavy-ion induced floating gate errors: LET and feature size dependence," IEEE Trans. Nucl. Sci., vol. 57, no. 4, pp. 1835-1841, Aug. 2010.

[26] J. G. A. Ausman and F. B. McLean, "Electron - hole pair creation energy in $\mathrm{SiO}_{2}$," Appl. Phys. Lett., vol. 26, no. 4, pp. 173-175, Feb. 1975.

[27] J. M. Benedetto and H. E. Boesch, "The relationship between ${ }^{60} \mathrm{Co}$ and $10 \mathrm{keV}$ x-ray damage in MOS devices," IEEE Trans. Nucl. Sci., vol. 33, no. 6, pp. 1317-1323, Dec. 1986.

[28] C. A. Klein, "Bandgap dependence and related features of radiation ionization energies in semiconductors," J. Appl. Phys., vol. 39, no. 4, pp. 2029-2038, Mar. 1968.

[29] R. C. Alig and S. Bloom, "Secondary-electron-escape probabilities," J. Appl. Phys., vol. 49, no. 6, pp. 34763480, Jun. 1978.

[30] Interplanetary Monitoring Platform (IMP-8).

Available:

http://spdf.gsfc.nasa.gov/imp8/project.html

[31] Advanced Composition Explorer (ACE). Available: http://www.srl.caltech.edu/ACE/

[32] J. A. Pellish, M. A. Xapsos, K. A. LaBel, P. W. Marshall, D. F. Heidel, K. P. Rodbell, M. C. Hakey, P. E. Dodd, M. R. Shaneyfelt, J. R. Schwank, R. C. Baumann, X. Deng, A. Marshal, B. D. Siearwski, J. D. Black, R. A. Reed, R. D. Schrimpf, H. S. Kim, M. D. Berg, M. J. Campola, M. R. Friendlich, C. E. Perez, A. M. Phan, and C. M. Seidleck, "Heavy Ion Testing at the Galactic Cosmic Ray Energy Peak," presented at the 10th European Conference on Radiation and Its Effects on Components and Systems, Brugge, Belgium, 2009.

[33] C. S. Dyer, K. Hunter, S. Clucas, D. Rodgers, A. Campbell, and S. Buchner, "Observation of solar particle events from CREDO and MPTB during the current solar maximum," IEEE Trans. Nucl. Sci., vol. 49, no. 6, pp. 2771-2775 Dec. 2002. 